\title{
Regularized super-resolution image reconstruction employing robust error norms
}

\author{
Antigoni Panagiotopoulou \\ Vassilis Anastassopoulos \\ University of Patras \\ Physics Department \\ Electronics Laboratory \\ Rio 26500, Greece \\ E-mail: vassilis@ physics.upatras.gr
}

\begin{abstract}
A high-resolution image is reconstructed from a sequence of subpixel shifted, aliased low-resolution frames, by means of stochastic regularized super-resolution (SR) image reconstruction. The Tukey $(T)$, Lorentzian $(\mathrm{L})$, and Huber $(\mathrm{H})$ cost functions are employed for the datafidelity term. The performance of the particular error norms, in SR image reconstruction, is presented. Actually, their employment in SR reconstruction is preceded by dilating and scaling their influence functions to make them as similar as possible. Thus, the direct comparison of these norms in rejecting outliers takes place. The bilateral total variation (BTV) regularization is incorporated as a priori knowledge about the solution. The outliers effect is significantly reduced, and the high-frequency edge structures of the reconstructed image are preserved. The proposed TTV, LTV, and HTV methods are directly compared with a former SR method that employs the $L_{1}$-norm in the data-fidelity term for synthesized and real sequences of frames. In the simulated experiments, noiseless frames as well as frames corrupted by salt-and-pepper noise are employed. Experimental results verify the robust statistics theory. Thus, the Tukey method performs best, while the $L_{1}$-norm technique performs inferiorly to the proposed techniques. () 2009 Society of Photo-Optical Instrumentation Engineers. [DOI: 10.1117/1.3265543]
\end{abstract}

Subject terms: super-resolution (SR); subpixel shift; aliasing; robust statistics; bilateral total variation (BTV).

Paper 090357R received May 18, 2009; revised manuscript received Sep. 10, 2009; accepted for publication Sep. 28, 2009; published online Nov. 19, 2009.

\section{Introduction}

Images of high-resolution (HR) are desired and often required in most electronic imaging applications. Employing signal processing techniques to create an HR image from multiple observed low-resolution (LR) ones is called superresolution (SR) image reconstruction. The information related to the relative displacement between the LR images is investigated, and spatial resolution is increased by integrating these images into a single image. Simultaneously, the effect of possible blurring and noise in the LR images is also removed. The most important advantage of the specific signal processing approaches is that they are much cheaper than HR imaging equipment. Furthermore, the existing LR imaging systems can still be utilized. ${ }^{1,2}$ Super-resolution image reconstruction methods ${ }^{3}$ produce more powerful resolution enhancement results than interpolation techniques that are often encountered in the literature. ${ }^{4,5}$

Several approaches to the problem of super-resolution image reconstruction have been developed. The categories of existing SR techniques 1 are presented in Table 1. Among these techniques, the regularized and ML-POCS hybrid are most advantageous. The stochastic regularized superresolution methods provide stable estimates effectively and distinguish between possible solutions by utilizing a priori image models. The choice of regularization or prior significantly affects the performance of SR algorithms. Table 2 presents the regularization techniques that are often em-

0091-3286/2009/\$25.00 @ 2009 SPIE ployed when performing the SR image reconstruction task. $^{6-9}$ The $L_{2}$-norm and Tikhonov techniques belong to the early efforts regarding regularization. In fact, the Tikhonov method is one of the most widely referenced regularization methods. In relation to the total variation technique, it is one of the most successful regularization techniques for denoising and deblurring. The bilateral total variation (BTV) method is a robust regularizer that is based on the spirit of TV criterion and a related technique named the bilateral filter. Several stochastic regularized approaches to SR image reconstruction are found in the literature. The $L_{1}$-norm estimator and the bilateral TV regularization are employed to deal with different data and noise models in Ref. 7 The particular method is robust to errors in motion and blur estimation while it preserves edges. Super-resolution image reconstruction by means of a texture prior is introduced in. Ref. 10 The $L_{2}$-norm estimator is employed for the data-fidelity term. The regularization takes the form of a probability density function that is based on sampled images. The Huber cost function serves for the data-fidelity term in. Ref. 11 In the same work, the Tikhonov and Huber-Tikhonov regularizations are also employed. In Ref. 12 , the $L_{1}$-norm measures the difference between the projected estimate of the HR image and each LR image, while the regularization takes the form of the bilateral TV prior. Moreover, a highly accurate registration algorithm, the fast affine block-based one, performs the registration task. In the SR reconstruction methods of Refs. 13 and 14, the fast affine block-based registration method is 
Table 1 Super-resolution image reconstruction approaches.

\begin{tabular}{|c|c|c|c|}
\hline SR approach & Advantages & Disadvantages & Comments \\
\hline \multirow[t]{2}{*}{ Nonuniform interpolation } & $\begin{array}{l}\text { Relatively low computa- } \\
\text { tional load }\end{array}$ & Limited degradation models & Most intuitive method (Ref. 1) \\
\hline & $\begin{array}{l}\text { Real-time applications are } \\
\text { possible }\end{array}$ & $\begin{array}{c}\text { Not guaranteed optimality of } \\
\text { the whole reconstruction } \\
\text { algorithm }\end{array}$ & \\
\hline \multirow[t]{2}{*}{ Frequency domain } & Theoretical simplicity & $\begin{array}{l}\text { Restriction to global transla- } \\
\text { tional motion and linear space } \\
\text { invariant (LSI) blur for the } \\
\text { observation model }\end{array}$ & $\begin{array}{l}\text { Makes explicit use of the } \\
\text { aliasing that exists in each LR } \\
\text { image (Ref. 1) }\end{array}$ \\
\hline & $\begin{array}{l}\text { Convenient for parallel im- } \\
\text { plementation }\end{array}$ & $\begin{array}{l}\text { Difficulty in applying the } \\
\text { spatial domain a priori } \\
\text { knowledge for regularization }\end{array}$ & \\
\hline \multirow[t]{2}{*}{ Regularized (stochastic) } & $\begin{array}{c}\text { The use of an edge-preserving } \\
\text { image prior } \\
\text { model }\end{array}$ & - & $\begin{array}{c}\text { Provides stable SR estimates } \\
\text { effectively; distinguishes } \\
\text { between possible solutions by } \\
\text { utilizing a priori image model } \\
\text { (Ref. 1) }\end{array}$ \\
\hline & $\begin{array}{l}\text { Robustness and flexibility in } \\
\text { modeling noise characteris- } \\
\text { tics and a priori knowledge } \\
\text { about the solution }\end{array}$ & & \\
\hline \multirow[t]{3}{*}{$\begin{array}{l}\text { Projection onto convex sets } \\
\text { (POCS) }\end{array}$} & Simplicity & No unique solution & $\begin{array}{l}\text { Alternative iterative approach } \\
\text { to incorporating prior knowl- } \\
\text { edge about the solution into }\end{array}$ \\
\hline & $\begin{array}{l}\text { Utilization of the powerful } \\
\text { spatial domain observation } \\
\text { model }\end{array}$ & Slow convergence & the reconstruction process (Ref. 1) \\
\hline & $\begin{array}{c}\text { Allowance for convenient } \\
\text { inclusion of a priori infor- } \\
\text { mation }\end{array}$ & High computational cost & \\
\hline $\begin{array}{c}\text { Maximum likelihood (ML)-POCS } \\
\text { hybrid }\end{array}$ & $\begin{array}{l}\text { All a priori knowledge is } \\
\text { effectively combined } \\
\text { Single optimal solution }\end{array}$ & - & $\begin{array}{l}\text { SR estimation by minimizing } \\
\text { the ML (or MAP) cost func- } \\
\text { tional while constraining the } \\
\text { solution within certain sets } \\
\text { (Ref. 1) }\end{array}$ \\
\hline \multirow[t]{3}{*}{ Iterative back-projection } & $\begin{array}{l}\text { Understood intuitively and } \\
\text { easily }\end{array}$ & No unique solution & $\begin{array}{l}\text { Similar to the back-projection } \\
\text { used in tomography (Ref. 1) }\end{array}$ \\
\hline & & $\begin{array}{l}\text { Difficulty in choosing the } \\
\text { back-projection kernel }\end{array}$ & \\
\hline & & $\begin{array}{l}\text { Difficulty in applying a priori } \\
\text { constraints }\end{array}$ & \\
\hline Adaptive filtering & - & - & $\begin{array}{l}\text { Based on adaptive filtering } \\
\text { theory applied on time axis } \\
\text { (Ref. 1) }\end{array}$ \\
\hline Motionless & - & - & $\begin{array}{l}\text { SR reconstruction from dif- } \\
\text { ferently blurred images with- } \\
\text { out relative motion (Ref. 1) }\end{array}$ \\
\hline
\end{tabular}

employed for motion estimation and the $L_{2}$-norm is used for the data-fidelity term. In Ref. 13 the BTV regularization compensates for the missing measurements information, whereas in Ref. 14, the Tikhonov regularization performs the particular task. Reference 15 introduces the Tukey norm for the data-fidelity cost function, while the Tikhonov regularization is added as a penalty term to the cost function. Reference 16 proposes the Lorentzian norm function for measuring the difference between the projected estimate of the HR image and each LR frame, while the regularization 
Table 2 Regularization techniques.

\begin{tabular}{|c|c|c|}
\hline Regularization technique & Mathematical form & Comments \\
\hline$L_{2}$-norm & $\|X\|_{2}^{2}$ & Promotes small energy (Ref. 6) \\
\hline Tikhonov & $\|\Gamma X\|_{2}^{2}$ & $\begin{array}{l}\text { Limits the total energy of the image } \\
\text { or forces spatial smoothness (Ref. } 7 \text { ) }\end{array}$ \\
\hline $\begin{array}{l}\text { Huber-Markov random field } \\
\text { (HMRF) }\end{array}$ & $A \exp \left\{-\frac{1}{k} \Sigma_{c \in C} \rho_{H b}\left(d_{c}^{t} X\right)\right\}$ & $\begin{array}{c}\text { The reconstruction relies on the } \\
\text { choice of the Huber function } \\
\text { parameter (Ref. 8) }\end{array}$ \\
\hline Lorentzian-Tikhonov & $\rho_{L r}(\Gamma X)$ & - \\
\hline Huber-Tikhonov & $\rho_{H b}(\Gamma X)$ & - \\
\hline Total variation & $\|\nabla X\|_{1}$ & $\begin{array}{c}\text { Penalizes the total amount of change } \\
\text { in the image as measured by the } \\
L_{1} \text {-norm of the magnitude of the } \\
\text { gradient (Ref. } 7 \text { ) }\end{array}$ \\
\hline Bilateral total variation & $\Sigma_{l=-P}^{P} \Sigma_{m=0}^{P} a^{|m|+|| \mid}\left\|X-S_{x}^{\prime} S_{y}^{m} X\right\|_{1}$ & $\begin{array}{l}\text { Based on the spirit of TV criterion } \\
\text { and a related technique called } \\
\text { bilateral filter (Ref. } 7 \text { ) }\end{array}$ \\
\hline Maximum entropy & Ref. 9 & $\begin{array}{l}\text { Produces sharp reconstructions of } \\
\text { point objects, such as star fields in } \\
\text { astronomical images (Ref. } 7 \text { ) }\end{array}$ \\
\hline Wavelet & $\|T X\|_{1}$ & $\begin{array}{l}\text { Wavelet basis decomposition of } \\
\text { measured data is applied (Ref. 6) }\end{array}$ \\
\hline
\end{tabular}

$\overline{X \text { high-resolution image; } \Gamma \text { high-pass operator; } k \text { tuning parameter of the density; } d_{c} \text { coefficient vector }}$ for clique $c$.

takes the form of the Tikhonov and Lorentzian-Tikhonov priors. The Huber error norm in combination with the BTV regularization is introduced in, Ref. 17 where experimentation is carried out employing thermal infrared data.

The present work belongs to the category of stochastic, typically Bayesian, regularized approaches to SR image reconstruction. Three new maximum a posteriori (MAP) estimation methods for performing the task of superresolution image reconstruction are presented. Namely, the Tukey error norm in combination with the BTV regularization (TTV), the Lorentzian error norm in combination with the BTV regularization (LTV) and the Huber norm combined with the BTV regularization (HTV) are the three methods that are proposed in the present work. The particular combinations of terms, for performing the task of SR image reconstruction, are novel. The performance of the aforementioned three error norms, in super-resolution reconstruction, is presented. In fact, the direct comparison of these estimators in rejecting outliers takes place, as they have been made as similar as possible through dilation and scaling of their influence functions. The results of the particular comparison verify the robust statistics theory. Therefore, an additional novel contribution of the present work is the direct comparison of the three estimators, Tukey, Lorentzian, and Huber, in performing SR image reconstruction, thus in rejecting outliers. An HR image is created from a sequence of subpixel-shifted, aliased LR frames. Simulated and real experiments are performed. In the simulated experiments, noiseless frames as well as noisy frames are employed, while resolution is increased by a factor of 4 . In the real experiment, resolution is enhanced by a factor of 3 . The results of the TTV, LTV, and HTV techniques are compared with those obtained by means of the SR technique, which is described in. Ref. ${ }^{7}$ The method in Ref. ${ }^{7}$ combines the $L_{1}$-norm with the BTV regularizer. According to the robust statistics theory, the Tukey error norm performs best in rejecting outliers, and the Lorentzian norm comes second in performance. The Huber error norm performs inferior to the Lorentzian norm and predominates over the $L_{1}$-norm. In the present work, experimentation verifies theory.

In Sec. 2 of this paper, the Tukey, Lorentzian, and Huber error norms are discussed. Section 3 describes the SR reconstruction, and Sec. 4 presents the experimental procedure regarding simulated experiments. The experimental results from employing synthesized sequences of frames are provided in Secs. 5 and 6. Section 7 presents the real SR experiment. Certain decisive aspects in relation to the present work are placed in Sec. 8, and conclusions are drawn in Sec. 9.

\section{Drawing Error Norms from Robust Statistics}

The field of robust statistics deals with estimation problems in which the data contains gross errors or outliers. ${ }^{18-20}$ Super-resolution image reconstruction is such an estimation problem. The desired estimate satisfies an optimization criterion where an objective function, named $\rho$-function, is minimized. The choice of the particular robust $\rho$-function or error norm is critical. To analyze the behavior of a given $\rho$-function, its influence function $\psi$-which is proportional to 
the derivative of $\rho$, is considered. The influence function characterizes the bias that a particular measurement has on the solution. For example, the quadratic $\rho$-function has a linear $\psi$-function. A least-squares estimate is very sensitive to outliers because the influence function increases linearly and without bound. The quadratic gives outliers too much influence. To increase robustness and reject outliers, the $\rho$-function must be more forgiving about outliers - that is, it should increase less rapidly than $x^{2}$.

An error norm from the robust statistics literature is the Huber norm. The Huber estimator is essentially the leastsquares estimator, but uses the $L_{1}$-norm for points that are considered outliers with respect to a certain threshold, which is called the Huber parameter. The linear growth for large $x$ makes approximation less sensitive to outliers. The Huber error norm and its influence function are given by

$\rho_{H b}(x)=\left\{\begin{array}{cc}x^{2}, & |x|<\text { hubpar } \\ 2 \text { hubpar }|x|-\text { hubpar }^{2}, & \text { otherwise }\end{array}\right.$,

$\psi_{H b}(x)=\left\{\begin{array}{cc}2 x, & |x|<\text { hubpar } \\ 2 \text { hubparsign }(x), & \text { otherwise }\end{array}\right.$,

where hubpar is the Huber parameter.

The influence curve of the Huber estimator is constant for all observations beyond a certain point. An $M$-estimator can be made more resistant by having the $\psi$-function, and hence the influence curve, return to 0 . Such an estimator is called a redescending estimator. Examination of a redescending influence function reveals that when the absolute value of $x$ increases beyond a fixed point, determined by the scale parameter, its influence is reduced. Outliers have diminishing effects on a redescending estimator. The Lorentzian error norm is such an estimator. However, its influence does not descend all the way to zero. A more robust norm that does descend to zero is the Tukey biweight error norm. The Lorentzian and Tukey biweight error norms and influence functions are as follows:

$\rho_{L r}(x)=\log \left[1+\frac{1}{2}\left(\frac{x}{\text { lorpar }}\right)^{2}\right]$,

$\psi_{L r}(x)=\frac{2 x}{2 \operatorname{lorpar}^{2}+x^{2}}$,

$\rho_{T k}(x)=\left\{\begin{array}{cc}\frac{x^{2}}{t_{k p a r^{2}}}-\frac{x^{4}}{t_{u k p a r^{4}}}+\frac{x^{6}}{3 t u k p a r^{6}}, & |x|<\text { tukpar } \\ \frac{1}{3}, & \text { otherwise }\end{array}\right.$,

$\psi_{T k}(x)=\left\{\begin{array}{c}\frac{2}{\text { tukpar }^{2}}\left\{x\left[1-\left(\frac{x}{\text { tukpar }}\right)^{2}\right]^{2}\right\}, \\ 0, \quad|x|<\text { tukpar } \\ \text { otherwise }\end{array}\right.$,

where lorpar and tukpar are the scale parameters. The pre-

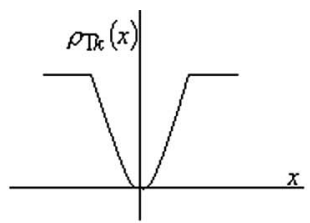

(a)

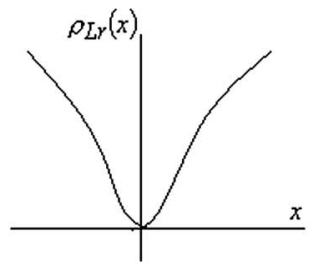

(c)

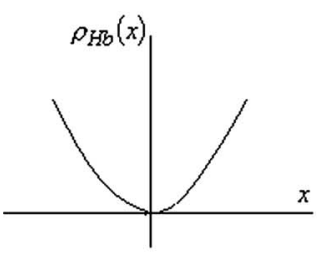

(e)

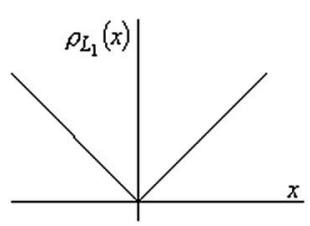

(g)

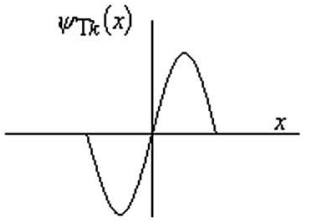

(b)

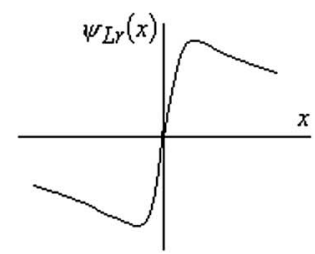

(d)

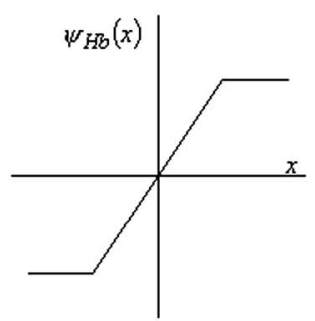

(f)

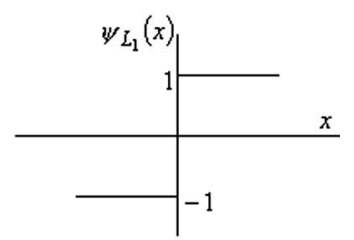

(h)
Fig. 1 The $\rho$-and $\psi$-functions of the various estimators. (a) Tukey biweight $\rho$-function. (b) Tukey biweight $\psi$-function. (c) Lorentzian $\rho$-function. (d) Lorentzian $\psi$-function. (e) Huber $\rho$-function. (f) Huber $\psi$-function. (g) $L_{1} \rho$-function. (h) $L_{1} \psi$-function.

ceding error norms and influence functions are plotted in Fig. 1.

The direct comparison of the prementioned three error norms can be performed after dilating and scaling their influence functions to make them as similar as possible. ${ }^{21}$ The aligned and scaled $\psi$-functions are plotted in Fig. 2. The Huber norm gives all outliers a constant weight of one, while the Tukey norm gives zero weight to outliers whose magnitude is above a certain value. The Lorentzian norm is between the other two. Based on the shape of the $\psi$-functions, we would expect super-resolution reconstruction employing the Tukey error norm to give better results than SR reconstruction employing the Lorentzian norm and both of them to predominate over super-resolution employing the Huber error norm. Accordingly, the $L_{1}$-norm estimation, which converges to median estimation, is expected to perform inferiorly to the prementioned three error norms. $^{17}$

\section{Super-Resolution Reconstruction}

\subsection{Data-Fidelity Term}

When dealing with the SR image reconstruction problem, the effect of outliers has to be reduced. Otherwise, errone- 


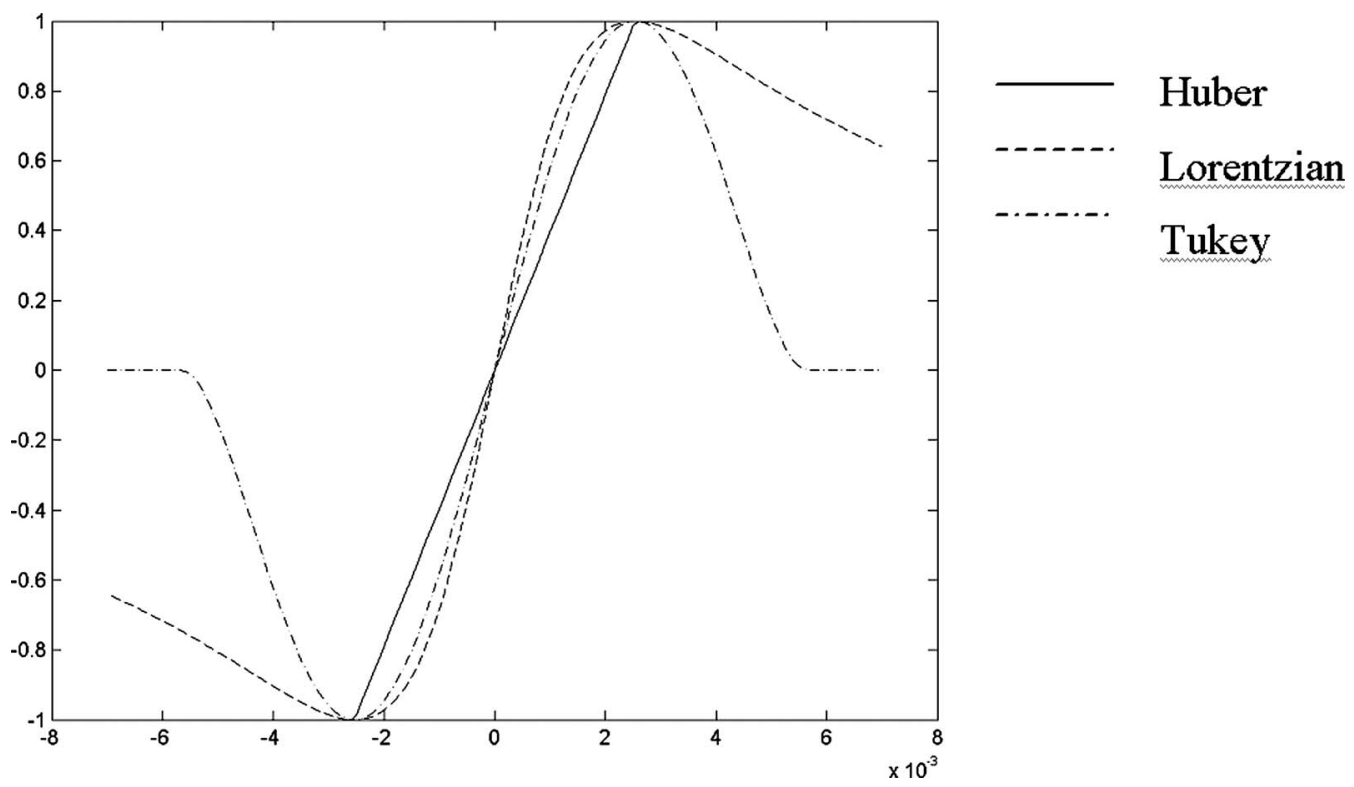

Fig. 2 The Tukey, Lorentzian, and Huber $\psi$-functions aligned and scaled. The direct comparison of the three error norms, in rejecting outliers, can now take place.

ous estimates will be obtained. ${ }^{22}$ Therefore, a robust estimator has to be employed for measuring the difference between the projected estimate of the HR image and each LR frame. In the present work, the Tukey, Lorentzian, and Huber estimators perform the particular task, and the performance of each of them is compared to that of the $L_{1}$-norm estimator.

The $T$ minimization criterion is formulated as follows:

$X_{T k}=\underset{X_{T k}}{\operatorname{ArgMin}}\left[\sum_{i=1}^{N} \rho_{T k}\left(D H F_{i} X_{T k}-Y_{i}\right)\right]$.

The $L$ minimization criterion is given by:

$X_{L r}=\underset{X_{L r}}{\operatorname{ArgMin}}\left[\sum_{i=1}^{N} \rho_{L r}\left(D H F_{i} X_{L r}-Y_{i}\right)\right]$.

As far as the $H$ minimization criterion is concerned:

$X_{H b}=\underset{X_{H b}}{\operatorname{ArgMin}}\left[\sum_{i=1}^{N} \rho_{H b}\left(D H F_{i} X_{H b}-Y_{i}\right)\right]$.

In the preceding relations, the analytic expression for $\rho_{T k}\left(D H F_{i} X_{T k}-Y_{i}\right), \quad \rho_{L r}\left(D H F_{i} X_{L r}-Y_{i}\right), \quad$ and $\rho_{H b}\left(D H F_{i} X_{H b}\right.$ $-Y_{i}$ ) can be formed using Eqs. (5), (3), and (1), respectively.

The operator $F_{i}, i=1,2, \ldots, N$, stands for translational motion among the LR frames. In fact, a motion vector that determines horizontal and vertical shift corresponds to each frame. The symbol $N$ presents the number of the available frames. Blurring is assumed to result from the same, spaceinvariant PSF that is represented by the operator $H$ regarding all frames. Furthermore, $D$ stands for the decimation that has been performed on the HR image to be acquired. The matrices $X_{T k}, X_{L r}$, and $X_{H b}$ denote the desired HR image, while $Y_{i}$ denotes the LR frames.

\subsection{Regularization Term}

Super-resolution image reconstruction is a numerically illposed problem. A kind of regularization must be included in the SR formulation to stabilize the problem or constrain the space of solutions. Additionally, regularization can serve for removing artifacts from the final answer and realizing considerable speedups in minimization. From a statistical perspective, as in the present work, regularization is incorporated as a priori knowledge about the solution. The HR image is not precisely known. The regularization offers some qualitative (a priori) knowledge in relation to this image. The particular knowledge could be some smoothness properties that the image should exhibit. Such a knowledge may be considered before the actual calculation of the HR estimate; hence, the name a priori knowledge. ${ }^{23}$

The bilateral TV regularizer is employed in the present paper. It is given by

$$
Y_{B T V}(X)=\sum_{l=-P}^{P} \sum_{m=0}^{P} a^{|m|+|l|}\left\|X-S_{x}^{l} S_{y}^{m} X\right\|_{1} .
$$

The operators $S_{x}^{l}$ and $S_{y}^{m}$ shift the image $X$ by $l$ and $m$ pixels horizontally and vertically, respectively, presenting several scales of derivatives all applied directly on the unknown image. The weight $a$ is a scalar taking values between 0 and 1 and gives a spatially decaying effect to the regularization terms summation. The parameter $P$ determines the size of the regularization kernel. The bilateral total variation regularization is based on the spirit of the total variation restoration model ${ }^{24}$ and the bilateral filter. ${ }^{25}$ The following two paragraphs give the theoretical background for the BTV regularization to provide some intuition as to why such a regularization is included in the super-resolution formulation of the present work.

Among all differential operators, the diffusion class is the most widely applied, in current image analysis, for non- 
linear denoising. In fact, anisotropic diffusion has attracted much attention. ${ }^{24}$ The TV anisotropic diffusion model is one of the most successful tools for image restoration, including both denoising and deblurring and edge enhancement. Unlike the conventional nonlinear filters, edges are quantitatively taken care of in this model. The particular model is deeply connected to functional analysis and geometry. Compared to the least-squares restoration models, the major difference of the total variation model is the minimization of the total variation-that is, the $L_{1}$-norm of the gradient, instead of the $L_{2}$-norm of the gradient: ${ }^{24}$

$T V[u]=\int_{\Omega}|\nabla u| \mathrm{d} x$.

Enormous gain emerges due to the transition to nonlinearity. Images are effectively restored, especially as far as edge preservation and enhancement is concerned.

The bilateral filter ${ }^{25}$ removes noise from images, keeping sharp edges. The particular filter is merely a weighted average of the local neighborhood samples, where the weights are computed based on spatial and radiometric distances between the center sample and the neighboring samples. The bilateral filter exploits all the relevant neighborhood in parallel. It does not apply some sort of diffusion of the neighborhood influence. Due to the use of several scales of derivatives, apart from penalizing smoothness with the first neighboring pixels, the penalization of nonsmoothness with distant neighbors also takes place. By applying the bilateral filter several times, the signal is smoothed and gets to a steady state.

\subsection{Super-Resolution Formulation}

Considering the above-mentioned data-fidelity and regularization terms, the SR problem is formulated. A gradientbased method, the steepest descent, is employed to perform the minimization task and results in $X$, the solution to the SR problem under consideration [Eqs. (12)-(14)]. The regularization term poses a penalty on the unknown matrix $X$ in order to direct it to a better formed solution. The coefficient $\lambda$ determines the strength with which the particular penalty is enforced and is called the regularization parameter. In relation to the parameter $\beta$, it is a scalar that determines the step size in the direction of the gradient. The constructed SR algorithms demonstrate reduced sensitivity to outliers:

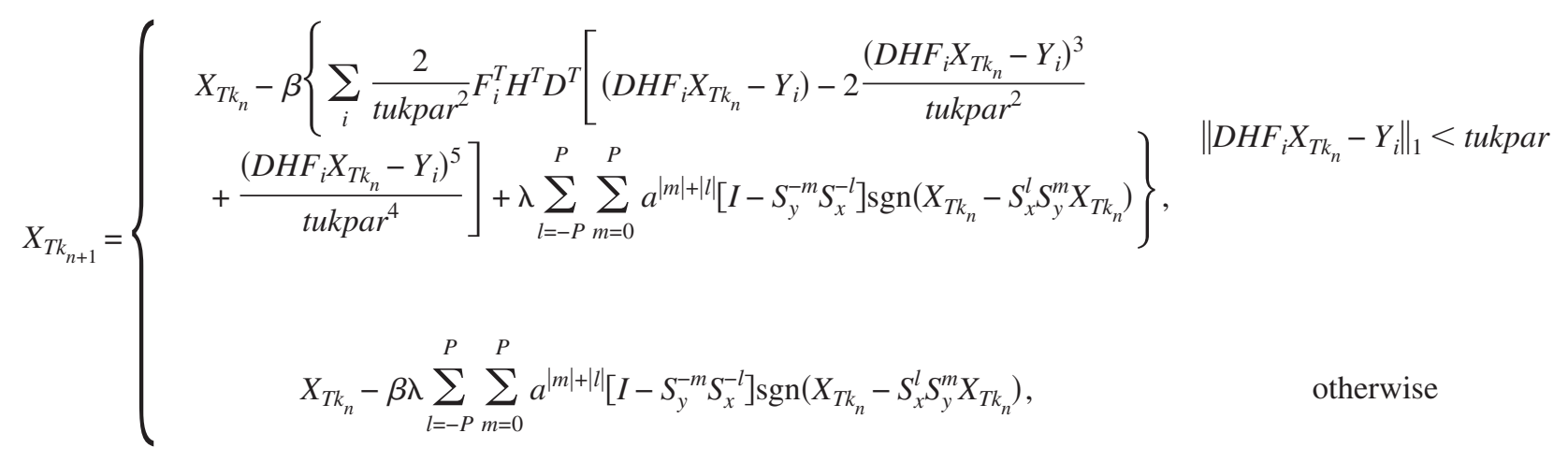

$\left\|D H F_{i} X_{T k_{n}}-Y_{i}\right\|_{1}<$ tukpar

$X_{L r_{n+1}}=X_{L r_{n}}-\beta\left\{\sum_{i} 2 F_{i}^{T} H^{T} D^{T} \frac{\left(D H F_{i} X_{L r_{n}}-Y_{i}\right)}{2 \operatorname{lorpar}^{2}+\left(D H F_{i} X_{L r_{n}}-Y_{i}\right)^{2}}+\lambda \sum_{l=-P}^{P} \sum_{m=0}^{P} a^{|m|+|l|}\left[I-S_{y}^{-m} S_{x}^{-l}\right] \operatorname{sgn}\left(X_{L r_{n}}-S_{x}^{l} S_{y}^{m} X_{L r_{n}}\right)\right\}$,

$X_{H b_{n+1}}=\left\{\begin{array}{c}X_{H b_{n}}-\beta\left\{\sum_{i} 2 F_{i}^{T} H^{T} D^{T}\left(D H F_{i} X_{H b_{n}}-Y_{i}\right)\right. \\ \left.+\lambda \sum_{l=-P}^{P} \sum_{m=0}^{P} a^{|m|+|l|}\left[I-S_{y}^{-m} S_{x}^{-l}\right] \operatorname{sgn}\left(X_{H b_{n}}-S_{x}^{l} S_{y}^{m} X_{H b_{n}}\right)\right\} \\ X_{H b_{n}}-\beta\left\{\sum_{i} 2 h u b p a r F_{i}^{T} H^{T} D^{T} \operatorname{sgn}\left(D H F_{i} X_{H b_{n}}-Y_{i}\right)\right. \\ +\lambda \sum_{l=-P} \sum_{m=0}^{P} a^{|m|+|l|}\left[I-S_{y}^{-m} S_{x}^{-l}\right] \operatorname{sgn}\left(X_{H b_{n}}-S_{x}^{l} S_{y}^{m} X_{H b_{n}}\right)\end{array}\right\}$

$\left\|D H F_{i} X_{H b_{n}}-Y_{i}\right\|_{1}<$ hubpar 
Table 3 The different SR problem formulations that are compared in the present paper along with their ranking (in descending order) in performance, as mandated by both visual and numerical comparisons at the conducted experiments.

\begin{tabular}{|c|c|c|c|}
\hline SR methods & Data-fidelity term & Regularization term & $\begin{array}{l}\text { Ranking in performance in al } \\
\text { cases of experimentation }\end{array}$ \\
\hline Method in Ref. 7 & $L_{1}$-norm & Bilateral TV & 4 \\
\hline TTV & Tukey norm & & 1 \\
\hline LTV & Lorentzian norm & & 2 \\
\hline HTV & Huber norm & & 3 \\
\hline
\end{tabular}

\section{Experimental Procedure-Simulated Experiments}

In Secs. (5) and (6), the performance of the resolution enhancement algorithms that are proposed in the present work is compared with that of an existing resolution enhancement method $^{7}$ in the case of employing synthesized sequences of frames (Table 3). The Tukey, Lorentzian, and Huber error norms, in combination with the BTV regularization, are proposed for performing the task of superresolution image reconstruction. The results obtained employing the prementioned three methods are compared with those coming from the super-resolution technique presented in Ref. 7. The particular technique employs the $L_{1}$-norm in the data-fidelity term and the bilateral TV regularizer in the regularization term. Table 3 also presents the ranking in performance for the various super-resolution methods, as mandated by visual and numerical comparisons at the conducted experiments, which follow in the next two sections. Actually, the robust statistics theory is verified. The particular performance ranking also holds for the real experiment presented in Sec. 7.

Several simulated experiments take place. At each experiment, super-resolution image reconstruction is applied to a synthesized LR sequence, which consists of 16 frames. Three different scenes are employed. A low-resolution image sequence is created from the original high-resolution image through the following procedure. At the beginning, in order to simulate the effect of camera PSF, the HR image is convolved with a symmetric Gaussian low-pass filter of size $4 \times 4$ and standard deviation equal to 1 . The image that results is then downsampled by the factor of 4 in each of the horizontal and vertical directions. The prementioned procedure, preceded by subpixel shifting the HR image in the vertical and horizontal directions employing various motion vectors, is followed to produce 15 LR images from the original HR image. Salt-and-pepper noise is also added to the LR frames. The experiments are implemented in MATLAB.

In the present work, the Tukey, Lorentzian, Huber, and $L_{1}$-error norms, in combination with the BTV regularization, are directly compared in performing the task of superresolution image reconstruction. The direct comparison of the Tukey, Lorentzian, and Huber error norms, through their influence functions, requires us to dilate and scale the functions to make them as similar as possible. Thus, first the values of the scale parameters hubpar, lorpar, and tuk- par are chosen to dilate each of the three influence functions in order to begin rejecting outliers at the same value par Ref. 21. We set hubpar=par, lorpar $=$ par $/ \sqrt{2}$, and tukpar $=\sqrt{5}$ par. Second, the three influence functions are scaled to return values in the same range. As far as the $L_{1}$-norm is concerned, there is no scale parameter involved. Regarding scaling, the particular influence function is scaled as all the rest ones.

When estimating the robust scale par of the image, a value for $D H F_{i} X-Y_{i}$ needs to be calculated. The value $m e$ dian $\left\{\right.$ median $\left.\left[a b s\left(D H F_{i} X-Y_{i}\right)\right]\right\}$ is considered instead, as $D H F_{i} X-Y_{i}$ is a matrix. Regarding each scene, three different HR images $-X \equiv$ original image; $X \equiv$ zeros; $X$ $\equiv$ bilinearly interpolated LR frame-are employed to calculate the value $D H F_{i} X-Y_{i}$ for each of the 16 frames. The 48 values that result are used to estimate the robust scale of the image. ${ }^{21}$ Actually, the specific estimation serves for giving an answer to the question how large the $D H F_{i} X-Y_{i}$ can be before we consider it to be an outlier. The robust scale values estimated for the various scenes, in the case of noiseless and noisy synthesized sequence of frames, are given in Table 4.

Table 5 presents the values of the parameter par that are employed by the TTV, LTV, and HTV techniques, for the various scenes, in cases of noiseless and noisy synthesized sequence of frames. Regarding the TTV technique, experimentation has been carried out with a value of par greater than that of the robust scale employed by the rest methods. The TTV technique exhibits a very low rhythm of reconstruction. The value of par affects the particular rhythm significantly, and it is increased to speed up the TTV reconstruction procedure. As results indicate, the employed value of par does not degrade the performance of the TTV technique. The values of the step size of the gradient, employed by the various methods for the three different scenes, are

Table 4 The "robust scales" estimated for the various scenes, in cases of noiseless and noisy synthesized sequence of frames.

\begin{tabular}{cccc}
\hline \hline Scene/data & EIA & Lena & Stanford \\
\hline Noiseless & 0.0010 & 0.0119 & 0.0267 \\
Noisy & 0.0015 & 0.0163 & 0.0326 \\
\hline \hline
\end{tabular}


Table 5 The parameter par employed by the TTV, LTV, and HTV methods for the various scenes, in cases of noiseless and noisy synthesized sequence of frames.

\begin{tabular}{cccc}
\hline \hline & EIA & $\begin{array}{c}\text { Lena } \\
\text { Scene/method }\end{array}$ & $\begin{array}{c}\text { Stanford } \\
\text { Noiseless/noisy }\end{array}$ \\
\hline TTV & $0.1 / 0.1$ & $0.05 / 0.05$ & $0.05 / 0.05$ \\
LTV & $0.0010 / 0.0015$ & $0.0119 / 0.0163$ & $0.0267 / 0.0326$ \\
HTV & $0.0010 / 0.0015$ & $0.0119 / 0.0163$ & $0.0267 / 0.0326$ \\
\hline \hline
\end{tabular}

given in Table 6 . Additionally, Table 6 presents the values of the regularization term parameters $a, \lambda$.

A good first approximation $X_{0}$ of the desired HR image is required, especially in the case of frames corrupted by noise. In the noiseless frames case, a bilinearly interpolated LR frame serves for the initialization $X_{0}$. In the noisy frames case, a bilinearly interpolated clean LR frame is set as initialization. The particular clean frame is created by applying a median estimation procedure at the noisy frames.

The computational cost of the tested SR methods, in terms of the number of iterations for convergence in cases of noiseless and noisy synthesized sequence of frames for the various scenes is presented in Table 7. The HTV method and the method in Ref. 7 exhibit the lowest computational cost. The TTV method computationally is the most demanding method. As mentioned earlier, this particular method exhibits a very low rhythm of reconstruction. Regarding the LTV method, the computational cost is between those of the TTV, HTV, and $L_{1}$-norm methods. The employed scene, as well as the employed value of the parameter par, affect the computational cost of a superresolution method. The EIA scene computationally is the most demanding scene. Notice that the particular scene also presents the lowest value of robust scale (Table 4). The Lena and Stanford scenes, whose robust scales are of the same order of magnitude, demand similar computational costs. In fact, these two scenes computationally are less demanding than the EIA scene.

\section{Experimental Results-Noiseless Synthesized Sequences of Frames}

\subsection{EIA Scene}

The original HR file, courtesy of Sina Farsiu, ${ }^{7}$ is shown in Fig. 3. The same figure depicts an LR frame of the synthe- sized EIA sequence. Figure 4 depicts parts of the SR image that results from implementing the method that employs the $L_{1}$-norm in the data-fidelity term and the BTV regularizer in the regularization term. The corresponding results of the proposed methods are demonstrated in the same figure. Visual comparison proves the predominance of the TTV, LTV, and HTV techniques over the method in Ref. 7. In fact, the TTV technique performs best. In the image resulting from this particular technique, the strippings are nearly perfectly reconstructed without any shadings. Additionally, the contour of the semifinished circles is exactly constructed, with no artifacts present. The same is true for all the depicted numerals, which are clearly legible. In descending order of performance, the TTV technique is first, the LTV second, and the HTV third. Table 8 contains numerical results regarding the predescribed comparison. The Xydeas and Petrovich, ${ }^{26}$ MSE, and correlation coefficient measures are in accordance with visual assessment as far as the various methods' performance is concerned. In fact, the robust statistics theory is verified.

\subsection{Lena Scene}

The original HR file, drawn from a website, is shown in Fig. 3. An LR frame of the synthesized Lena sequence is depicted in the same figure. Figure 4 depicts parts of the SR images that result from implementing the proposed superresolution methods. The corresponding results of the method that employs the $L_{1}$-norm in the data-fidelity term and the bilateral TV regularizer in the regularization term are demonstrated in the same figure. Visual comparison asserts that the TTV, LTV, and HTV techniques predominate over the method in Ref. 7. Again, the TTV technique performs best. In the image resulting from the TTV technique, the outline of the front part of the hat is refined and does not exhibit any discontinuities, which indicates detailed reconstruction and high resolution. The same is true for the line connecting the noise to the eyebrow at the left. Additionally, the eye pupil has been reconstructed in detail. Inspecting visually the prementioned details in the remaining SR reconstructed images, we conclude that super-resolution performance degrades from the TTV to the LTV, HTV, and $L_{1}$-norm techniques. In descending order of performance, the TTV technique is first, the LTV second, and the HTV third. The $L_{1}$-norm technique is inferior to all the others. Numerical results regarding the predescribed comparison are presented in Table 9. The Xydeas and Petrovich, MSE, and correlation coefficient measures are in accordance with

Table 6 The parameters $\beta, a$, and $\lambda$ employed by the tested methods for the various scenes, in cases of both noiseless and noisy synthesized sequence of frames.

\begin{tabular}{ccccc}
\hline \hline Method/parameter & $\begin{array}{c}\text { TTV } \\
\text { EIA/Lena/Stanford }\end{array}$ & $\begin{array}{c}\text { LTV } \\
\text { EIA/Lena/Stanford }\end{array}$ & $\begin{array}{c}\text { HTV } \\
\text { EIA/Lena/Stanford }\end{array}$ & $\begin{array}{c}\text { Method in Ref. 7 } \\
\text { EIA/Lena/Stanford }\end{array}$ \\
\hline$\beta$ & $0.04 / 0.04 / 0.04$ & $0.008 / 0.02 / 0.02$ & $0.02 / 0.02 / 0.02$ & $0.01 / 0.005 / 0.005$ \\
$a$ & $0.01 / 0.04 / 0.01$ & $0.01 / 0.04 / 0.01$ & $0.01 / 0.04 / 0.01$ & $0.01 / 0.04 / 0.01$ \\
$\lambda$ & $0.003 / 0.009 / 0.003$ & $0.003 / 0.009 / 0.003$ & $0.003 / 0.009 / 0.003$ & $0.003 / 0.009 / 0.003$ \\
\hline
\end{tabular}



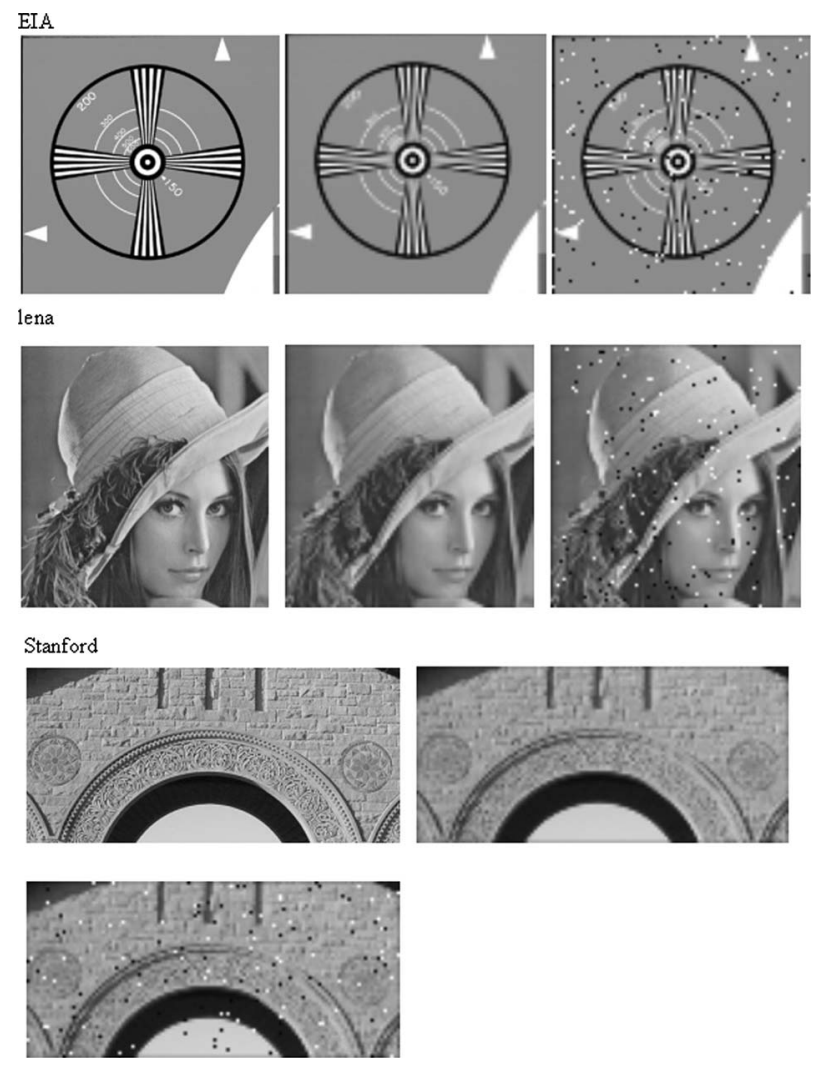

Fig. 3 The original HR image along with the noiseless and the corrupted (by salt-and-pepper noise) LR frame for the EIA, Lena, and Stanford scenes.

visual assessment with regard to the various methods performance. Concluding, visual and numerical assessments verify the robust statistics theory.

\subsection{Stanford Scene}

Figure 3 depicts the original HR file, which has been drawn from a website. ${ }^{27}$ An LR frame of the synthesized Stanford sequence is depicted in the same figure. Parts of the images obtained by means of the proposed super-resolution methods are shown in Fig. 4. The corresponding results of the method that employs the $L_{1}$-norm in combination with the bilateral TV regularizer are demonstrated in the same figure. According to visual inspection, the TTV, LTV, and

Table 7 The number of iterations for convergence regarding the tested SR methods, in cases of noiseless and noisy synthesized sequence of frames, for the various scenes.

\begin{tabular}{cccc}
\hline \hline $\begin{array}{c}\text { Scene/ } \\
\text { method }\end{array}$ & $\begin{array}{c}\text { EIA } \\
\text { Noiseless/ } \\
\text { noisy }\end{array}$ & $\begin{array}{c}\text { Lena } \\
\text { Noiseless/ } \\
\text { noisy }\end{array}$ & $\begin{array}{c}\text { Stanford } \\
\text { Noiseless/ } \\
\text { noisy }\end{array}$ \\
\hline TTV & $850 / 850$ & $400 / 400$ & $401 / 400$ \\
LTV & $16750 / 11750$ & $200 / 200$ & $200 / 200$ \\
HTV & $601 / 501$ & $20 / 20$ & $100 / 100$ \\
Method in Ref. 7 & $400 / 400$ & $40 / 40$ & $150 / 150$ \\
\hline \hline
\end{tabular}
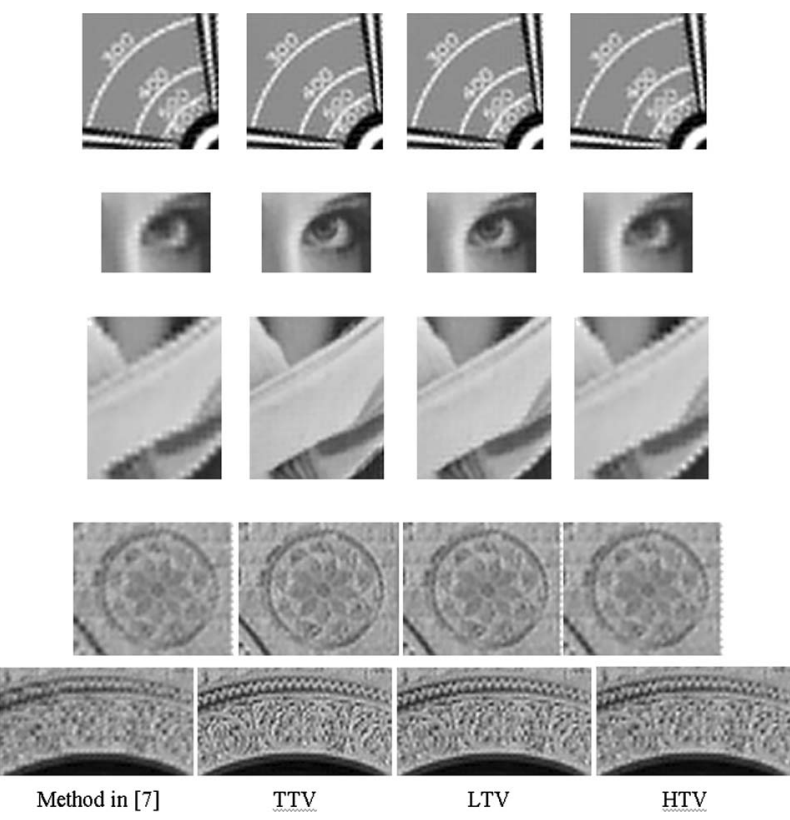

Fig. 4 Parts of the SR reconstructed images, resulting from the various SR methods, in the case of noiseless synthesized sequence of frames. Here, differences among the differently constructed HR images are discernible. The TTV technique exhibits the best performance. The LTV technique performs inferiorly to the TTV but superior to the HTV technique. The method in Ref. 7 performs inferior to all the other methods.

HTV techniques predominate over the method in Ref. 7. Actually, the TTV technique exhibits the best performance. In the image resulting from the TTV technique, the details of the carving that decorates the arched entrance are discernible, the two anaglyph patterns that look like flowers have been exactly reconstructed, while each stretcher of the wall discriminates from all the others. Focusing on the particular characteristics, it is obvious that the LTV technique performs inferiorly to the TTV and predominates over the HTV technique. The Huber norm technique performs better than the method in Ref. 7. In descending order of performance, the TTV technique is first, the LTV second, and the HTV third. The $L_{1}$-norm technique performs inferiorly to all the others. Numerical results regarding the predescribed comparison are presented in Table 10. The Xydeas and Petrovich, MSE, and correlation coefficient measures are in accordance with visual assessment in relation to the various methods' performance. Thus, the robust statistics theory is verified by both visual and numerical assessments.

Table 8 Numerical results regarding the EIA scene in the case of noiseless synthesized sequence of frames.

\begin{tabular}{ccccc}
\hline \hline Method/measure & TTV & LTV & HTV & Method in Ref. 7 \\
\hline Xydeas and Petrovich & 0.8893 & 0.8801 & 0.8534 & 0.8506 \\
MSE & 0.0033 & 0.0056 & 0.0068 & 0.0069 \\
Correlation coefficient & 0.9633 & 0.9392 & 0.9272 & 0.9245 \\
\hline
\end{tabular}


Table 9 Numerical results regarding the Lena scene in the case of noiseless synthesized sequence of frames.

\begin{tabular}{ccccc}
\hline \hline Method/measure & TTV & LTV & HTV & Method in Ref. 7 \\
\hline Xydeas and Petrovich & 0.9331 & 0.9203 & 0.8870 & 0.8805 \\
MSE & 0.0007 & 0.0012 & 0.0016 & 0.0017 \\
Correlation coefficient & 0.9901 & 0.9840 & 0.9772 & 0.9761 \\
\hline \hline
\end{tabular}

\section{Experimental Results-Noisy Synthesized Sequences of Frames}

\subsection{EIA Scene}

The experiment is conducted by adding salt-and-pepper noise of density $D=0.02$ to each frame, as well. A LR frame of the EIA sequence, corrupted by noise, is shown in Fig. 3. Parts of the HR images that result from the various super-resolution methods, when salt-and-pepper noise is present at the LR frames, are demonstrated in Fig. 5. Visual inspection asserts that all the methods have performed resolution enhancement as well as noise removal. Small portions of noise are discernible only at the gray background of the SR reconstructed image that results from the HTV technique. This can be attributed to the quadratic form of the Huber estimator. Table 11 presents numerical results as far as the noisy simulated experiment is concerned. Regarding PSNR, we use the definition

$$
\operatorname{PSNR}_{(d B)}=10 \log _{10} \frac{g_{f_{s}}^{2}}{M S E+\frac{1}{12}},
$$

where $g_{f_{s}}$ is the maximum pixel value of the original image. The TTV technique performs best; the LTV technique is inferior to the TTV and superior to the HTV technique. The technique in Ref. 7 is inferior to all the other techniques. Thus, experimental results are in accordance with the robust statistics theory as far as the various norms performance is concerned.

\subsection{Lena Scene}

Experimentation is carried out by adding salt-and-pepper noise of density $D=0.02$ to each frame of the Lena sequence as well. An LR Lena frame, corrupted by noise, is shown in Fig. 3. Figure 5 demonstrates parts of the HR images that result from the various super-resolution methods when salt-and-pepper noise is present at the LR frames.

Table 10 Numerical results regarding the Stanford scene in the case of noiseless synthesized sequence of frames.

\begin{tabular}{ccccc}
\hline \hline Method/measure & TTV & LTV & HTV & Method in Ref. 7 \\
\hline Xydeas and Petrovich & 0.8869 & 0.8740 & 0.8491 & 0.8337 \\
MSE & 0.0061 & 0.0084 & 0.0097 & 0.0098 \\
Correlation coefficient & 0.9363 & 0.9115 & 0.8971 & 0.8950 \\
\hline
\end{tabular}
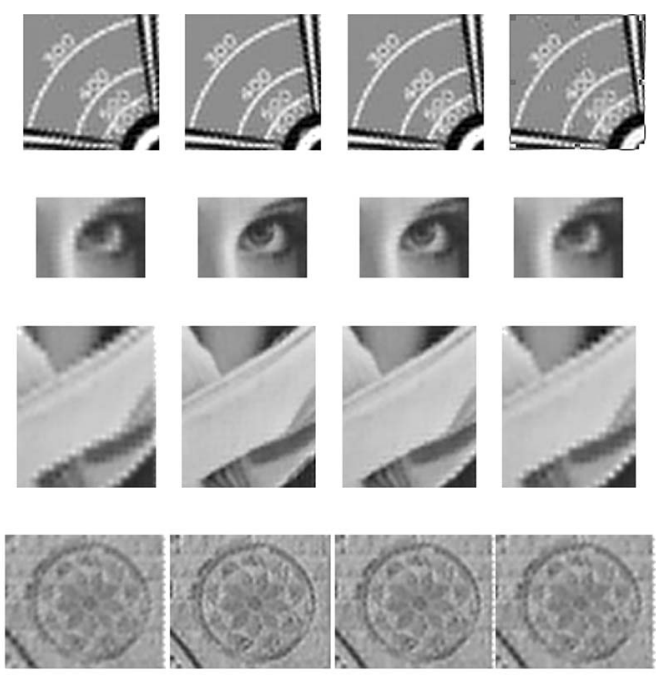

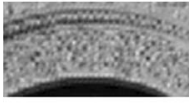

Method in [7]

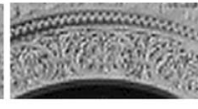

TTV

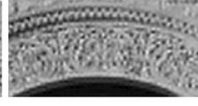

LTV

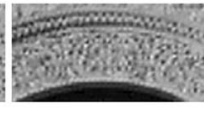

HTV
Fig. 5 Parts of the SR reconstructed images, resulting from the various SR methods, in the case of synthesized sequence of frames corrupted by salt-and-pepper noise. Here, differences among the differently constructed HR images are discernible. All the methods have performed resolution enhancement as well as noise removal. Small portions of noise are discernible only at the EIA gray background, which results from the HTV technique. This can be attributed to the quadratic form of the Huber estimator. The TTV technique performs best. The LTV and HTV techniques are second and third, respectively, in performance. The method in Ref. 7 performs inferiorly to all the other methods.

According to visual inspection, all the methods have performed resolution enhancement as well as noise removal. In fact, there are no portions of noise discernible anywhere. Table 12 presents numerical results with regard to the noisy simulated experiment. The TTV technique performs best, the LTV technique is second in performance, and the HTV technique third. The technique in Ref. 7 performs inferiorly to all the other techniques. Thus, the robust statistics theory is verified.

\subsection{Stanford Scene}

The experiment is also conducted by adding salt-andpepper noise, $D=0.02$, to the Stanford sequence of frames.

Table 11 Numerical results regarding the EIA scene in the case of synthesized sequence of frames corrupted by salt-and-pepper noise.

\begin{tabular}{ccccc}
\hline \hline Method/measure & TTV & LTV & HTV & Method in Ref. 7 \\
\hline Xydeas and Petrovich & 0.8831 & 0.8739 & 0.8514 & 0.8453 \\
MSE & 0.0037 & 0.0057 & 0.0070 & 0.0071 \\
Correlation coefficient & 0.9594 & 0.9374 & 0.9250 & 0.9215 \\
PSNR & 10.6031 & 10.5045 & 10.4415 & 10.4367 \\
\hline \hline
\end{tabular}


Table 12 Numerical results regarding the Lena scene in the case of synthesized sequence of frames corrupted by salt-and-pepper noise.

\begin{tabular}{ccccc}
\hline \hline Method/measure & TTV & LTV & HTV & Method in Ref. 7 \\
\hline Xydeas and Petrovich & 0.9314 & 0.9136 & 0.8817 & 0.8784 \\
MSE & 0.0008 & 0.0011 & 0.0017 & 0.0018 \\
Correlation coefficient & 0.9895 & 0.9850 & 0.9759 & 0.9750 \\
PSNR & 10.4028 & 10.3874 & 10.3566 & 10.3515 \\
\hline \hline
\end{tabular}

Figure 3 depicts an LR Stanford frame that is corrupted by noise. Parts of the HR images that result from the various super-resolution methods, in the noisy frames case, are shown in Fig. 5. Visual inspection asserts that all the methods have performed resolution enhancement and noise removal. Actually, there are no portions of noise discernible anywhere. Numerical results concerning the noisy simulated experiment are given in Table 13. The TTV technique performs best in rejecting outliers; the LTV technique is inferior to the TTV and superior to the HTV technique. The technique in Ref. ${ }^{7}$ exhibits the worst performance. Concequently, experimental results verify the robust statistics theory.

\section{Experiment Employing Real Sequence of Frames}

In the present work, experimentation is also carried out employing a real sequence of frames. The Tukey, Lorentzian, and Huber error norms, in combination with the BTV regularization, are proposed for performing superresolution image reconstruction by a resolution enhancement factor of 3. The SR performance of the proposed techniques is compared with that of the technique presented in Ref. 7, which employs the $L_{1}$-norm in combination with the BTV regularization. The results of the real experiment verify the robust statistics theory. Thus, the TTV method comes first in performance, the LTV method second, and the HTV method third. The $L_{1}$-norm technique performs inferiorly to the HTV method. The direct comparison of these methods in performing the task of SR image reconstruction takes place.

The employed sequence of frames was drawn from Peyman Milanfar's web page. ${ }^{7}$ The particular real-image se-

Table 13 Numerical results regarding the Stanford scene in the case of synthesized sequence of frames corrupted by salt-andpepper noise.

\begin{tabular}{ccccc}
\hline \hline Method/measure & TTV & LTV & HTV & Method in Ref. 7 \\
\hline Xydeas and Petrovich & 0.8863 & 0.8702 & 0.8455 & 0.8308 \\
MSE & 0.0062 & 0.0086 & 0.0099 & 0.0100 \\
Correlation coefficient & 0.9355 & 0.9098 & 0.8956 & 0.8935 \\
PSNR & 10.2733 & 10.1585 & 10.0975 & 10.0928 \\
\hline \hline
\end{tabular}

Table 14 The parameters par, $\beta$, a, and $\lambda$ employed by the tested methods in the case of the real alpaca sequence of frames.

\begin{tabular}{ccccc}
\hline \hline Method/parameter & TTV & LTV & HTV & Method in Ref. 7 \\
\hline par & 40 & 10 & 10 & - \\
$\beta$ & 10 & 5 & 5 & 5 \\
$a$ & 0.18 & 0.18 & 0.18 & 0.18 \\
$\lambda$ & 0.1 & 0.1 & 0.1 & 0.1 \\
\hline \hline
\end{tabular}

quence was captured with a 3COM camera, Model No. 3718. Motion from two separate sources is present in this sequence. In fact, by shaking the camera, a global motion was created for each individual frame. Also, an alpaca statue was independently moved in 10 of the 55 frames of the sequence. In the SR image reconstruction task employing the real alpaca sequence, motion estimation is performed by means of the MDSP resolution enhancement software. ${ }^{28}$ Regarding the unknown camera PSF, a $3 \times 3$ Gaussian kernel with standard deviation equal to 1 is considered.

The values of the parameter par that are employed by the TTV, LTV, and HTV methods are presented in Table 14. The LTV and HTV methods employ the theoretical value, robust scale, par $=10$. Nevertheless, the TTV method requires the greater value $p a r=40$ as an outliers rejection threshold for performing the SR image reconstruction task. The values of the step size of the gradient as well as the common values of the regularization term parameters $a, \lambda$ for the various methods are also given in Table 14. The parameter values employed in the real SR experiment are of a different order of magnitude than those employed in the simulated SR experiments and presented in Tables 5 and 6. This happens because the real alpaca data exhibit a different range of values than the synthesized EIA, Lena, and Stanford data. As far as the first approximation X0 of the desired HR image is concerned, a bilinearly interpolated LR frame is considered. With regard to the number of iterations for convergence, the TTV technique requires 20 iterations. The LTV and HTV techniques require 75 and 50 iterations, correspondingly. The $L_{1}$-norm technique needs 200 iterations to converge.

Two frames of the real LR sequence are demonstrated in Fig. 6. The same figure depicts the SR image that results from the technique that employs the $L_{1}$-norm in the datafidelity term and the BTV regularizer in the regularization term. The corresponding results of the proposed techniques are also shown in Fig. 6. Visual comparison asserts that the TTV, LTV, and HTV methods predominate over the method in Ref. 7. Actually, the TTV technique performs best. In the image resulting from this technique, the dcor at the left and bottom sides of the vase, the schematism at the center of the vase, the arrow at the right, the outline of the alpaca neck, as well as the alpaca tail are exactly constructed with no artifacts present. Visual inspection of the prementioned details in the remaning SR reconstructed images asserts that super-resolution performance degrades from the TTV to the LTV, HTV, and $L_{1}$-norm techniques. There is no original 
Method in [7]

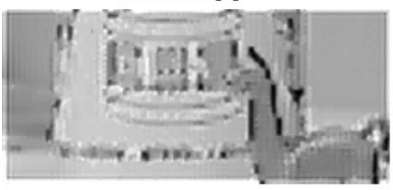

LTV

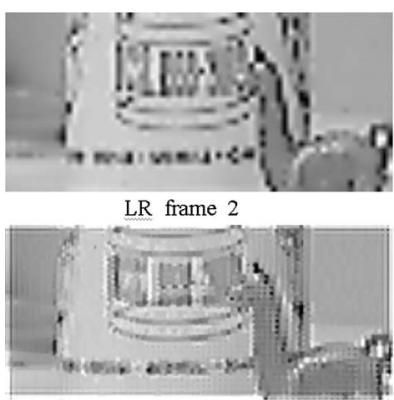

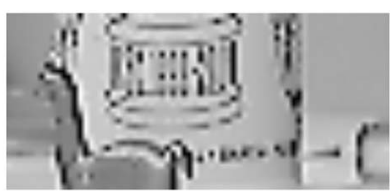

LR frame 53

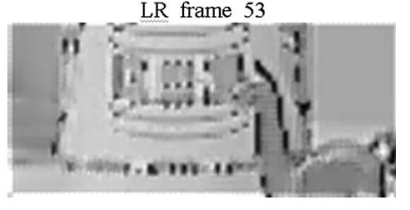

TTV

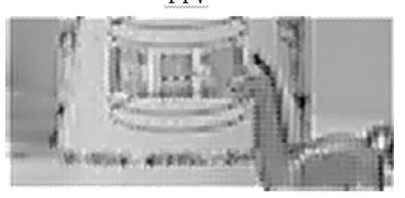

HTV
Fig. 6 The SR reconstructed images, resulting from the various SR methods, in the case of employing the real alpaca sequence of frames. Frames 2 and 53 of the LR sequence are also demonstrated. Differences among the differently constructed HR images are discernible. The TTV technique performs best. The LTV and HTV techniques are second and third, respectively, in performance. The method in Ref. 7 performs inferiorly to the HTV method.

HR image available to conduct numerical comparisons. In descending order of performance, the TTV technique is first, the LTV second, and the HTV third. The $L_{1}$-norm technique performs inferiorly to the other techniques. Concluding, the experimental results verify the robust statistics theory.

In accordance with the simulated experiments, the results of the real experiment verify the robust statistics theory. However, there are inherent difficulties in performing super-resolution image reconstruction employing a real sequence of frames, in contradiction with the simulated SR experiments. Actually, in the real experiment, the terms of the image acquisition model approximate the actual image formation. Nevertheless, in the simulated experiments, the image acquisition modeling terms capture the actual effects of the image formation process. Therefore, the overall super-resolution performance in the simulated experiments is much better than that in the real experiment. Incorrect approximations in the image acquisition model can lead to significant reduction in overall SR image reconstruction performance. In fact, the performance of motion estimation is of paramount importance to the performance of superresolution image reconstruction.

\section{Discussion}

The differences in the SR performances of the TTV, LTV, and HTV methods can be explained in terms of the intrinsic differences between the forms of the error norms employed for the data-fidelity term. Specifically, the Tukey norm gives zero weight to outliers whose magnitude is above a certain value. Therefore, certain outliers are discarded by the Tukey norm. The remaining outlier are given weights of decreasing values according to their magnitude. With regard to the Huber norm, it gives all outliers a constant weight of one. Thus, the Huber norm treats all outliers equally and does not discard any of them. As far as the treatment of outliers performed by the Lorentzian norm is concerned, it stands between the aforementioned outlier treatments. The Tukey estimator performs best in rejecting outliers. Accordingly, due to the Tukey estimator superior performance in the treatment of outliers, the TTV method results in high-resolution images with no artifacts present and exact reconstruction even of the finest details, for synthesized and real sequences of frames. The LTV and HTV methods are second and third, respectively, in SR image reconstruction performance as determined by their treatment of outliers. The particular ranking in methods performance holds for all scenes with which experimentation has been carried out and is consistent with the estimators' theoretical performance.

In the presented experiments, the parameters to deal with are $\beta$, par, $a$, and $\lambda$. Each method requires a different value for the parameter $\beta$. The particular parameter determines the step size in the direction of the gradient and is closely related to the number of iterations for convergence. The parameter par has a common value for the LTV and HTV methods. In order to avoid an extremely slow reconstruction procedure, the TTV method employs a value of par greater than that employed by the LTV and HTV methods. Actually, the rejection of outliers begins at the particular value par. The regularization term parameters $a, \lambda$ have common value for all methods. The parameter $\lambda$ determines the strength of the imposed penalty on the unknown matrix $X$, while $a$ gives a spatially decaying effect to the regularization terms summation. Earlier works ${ }^{7,16}$ have performed comparison of SR methods that employ the BTV regularizer, retaining common value for the parameter $a$ but changing the value of the parameter $\lambda$ from one method to another. Nevertheless, experimentation shows that the direct comparison strictly demands common values for the parameters $a, \lambda$.

In the present work, four different SR reconstruction methods are tested. There is no similarity among these methods as far as the behavior in the reconstructionconvergence procedure is concerned. The step size $\beta$ and the number of iterations for convergence characterize each super-resolution method. In fact, $\beta$ and the number of iterations for convergence are conversely proportional, referring to the values of $\beta$ that are appropriate for convergence. With regard to the latter notice, experimentation proves that there is more than one value of $\beta$ for which a method converges to the desired answer. Actually, for each method, there exists an upper value of $\beta$ over which the search for the global minimum is of no success. Over the entire range of appropriate $\beta$ values, the value that gives a reconstruction procedure both fast and exhibiting less oscillatory movements is chosen. Thus, choosing the appropriate value of $\beta$ requires visually monitoring the image outcome of all iterations and is not based only on the outcome of the final iteration.

Furthermore, the experimentation carried out shows that it is difficult to formulate an automatic/semiautomatic parameter estimation procedure. Actually, the value of the parameter par has to be estimated by means of standard techniques that perform estimation of the outliers rejection threshold. As far as the parameters $\beta, a$, and $\lambda$ are concerned, their values have to be specified manually through visual monitoring. 
Apart from visual inspection, the three numerical measures Xydeas and Petrovich, MSE, and correlation coefficient are employed to assess the super-resolution performance of each method. The Xydeas and Petrovich measure $^{26}$ is an objective test of edge information between two images. It measures the commonality of edge information between the original and the reconstructed image. The particular measure takes values in the range $[0,1]$. Its value is 0 when the images under comparison do not share any edge information, whereas its value is 1 when there is no loss of edge information from the original image to the reconstructed image. The MSE is the most popular error function, and its value should be as small as possible to have desired performance. With regard to the correlation coefficient measure, it gives the correlation coefficient between the reconstructed and the original image. In the ideal case, its value equals 1 . Through experimentation, it is concluded that when the correlation coefficient measure gives a value greater than that of the Xydeas and Petrovich measure, the corresponding image should be visually pleasant.

After having obtained the best final SR outcome of each method, the direct comparison of the various methods performance takes place. Through experimentation, it is concluded that the end in the super-resolution reconstruction procedure is an ambiguous sense. According to the Xydeas and Petrovich numerical measure, as reconstruction iterations go by, resolution enhancement is achieved even when the image visually remains unchanged. In detail, for quite a large number of iterations, an increase in this measure value, per the fourth decimal digit, takes place. The iteration, before resolution enhancement gets perceived per the fourth decimal digit of the Xydeas and Petrovich measure, is considered the final iteration of reconstruction for each method. It has been observed that each method exhibits a special rhythm of reconstruction. The particular rhythm is closely related to the number of iterations each method needs to complete the super-resolution image reconstruction.

Additionally, for direct comparison purposes, the $\psi$-functions of the Tukey, Lorentzian, Huber, and $L_{1}$ estimators are scaled to return values in the same range. The particular scaling causes a slight fall in each method's performance. Nevertheless, performing this scaling is unavoidable.

\section{Conclusions}

In this work, a high-resolution image is created from subpixel-shifted, aliased LR frames by means of stochastic regularized SR image reconstruction. Simulated and real experiments are conducted. In the simulated experiments, resolution is enhanced by a factor of 4 . In the real experiment, the resolution enhancement factor equals 3 . Three super-resolution methods are presented. The Tukey, Lorentzian, and Huber norms are employed for the data-fidelity term. The particular error norms demonstrate robustness in the presence of outliers. Regularization takes the form of the bilateral TV regularizer and compensates for the missing measurements information. In this work, the performance of Tukey, Lorentzian, and Huber estimators in super-resolution image reconstruction is presented. Actually, their utilization in the task of super-resolution reconstruction is preceded by dilating and scaling their influence functions to make them as similar as possible. Therefore, the particular three estimators are directly compared as far as rejection of outliers is concerned. The comparison carried out assesses in accordance with the robust statistics theory. The performance of the proposed TTV, LTV, and HTV methods is directly compared with that of a former regularized SR technique. In the case of synthesized sequences of frames as well as in the case of real sequence of frames, experimental results verify the robust statistics theory. Thus, the TTV technique performs best, and the LTV technique predominates over the HTV method, while the existing $L_{1}$-norm method performs inferiorly to all the other methods.

The Tukey, Lorentzian, and Huber estimators cope with the SR image reconstruction problem in the case of synthesized sequences of frames as well as in the case of real sequence of frames for various scenes. A key component of these estimators is the scale parameter, which controls the rejection of outliers. The value of this parameter is defined with respect to the robust scale of the image. Actually, the particular value varies with respect to the scene and the presence or absence of noise in the LR sequence of frames. Thus, the scale parameter reinforces the effective treatment of outliers for various cases of data. Additionally, the number of iterations for convergence characterizes each SR method. Through experimentation, it is concluded that the type of the scene, which is closely related to the value of the scale parameter, affects the rate of convergence of a particular method. Actually, an increased value of the scale parameter leads to a faster reconstruction-convergence procedure.

In the simulated experiments conducted in the present work, in addition to employing noiseless frames, noisy experimentation has been also carried out considering only salt-and-pepper noise. This particular type of noise is a representative one, as it fills the image with outliers. Experimentation with different noise models, such as Gaussian or speckle, could also take place. The latter type of experimentation might lead to a ranking in the performance of the tested methods different from that obtained in the present work. In fact, the relation between the model of the distributional characteristic of the noise and the model of the employed estimator could affect the SR reconstruction performance.

\section{References}

1. S. C. Park, M. K. Park, and M. G. Kang, "Super-resolution image reconstruction: a technical overview," IEEE Signal Process. Mag. 20(3), 21-36 (2003).

2. S. Chaudhuri, Super-Resolution Imaging, Kluwer Academic Publishers, Boston, MA (2001).

3. A. Panagiotopoulou and V. Anastassopoulos, "Super-resolution image reconstruction employing Kriging interpolation technique," in Proc. IWSSIP 2007 \& EC-SIPMCS 2007, Maribor, Slovenia, pp 151-154 (2007).

4. V. Tsagaris, A. Panagiotopoulou, and V. Anastassopoulos, "Interpolation in multispectral data using neural networks," Proc. SPIE 5573, 460-470 (2004).

5. A. Panagiotopoulou and V. Anastassopoulos, "Scanned images resolution improvement using neural networks," Neural Comput. Appl. 17(1), 39-47 (2008)

6. M. Elad, "Example-based prior for super-resolution," in Int. Conf. Super-Resolution Imaging (2005).

7. S. Farsiu, M. D. Robinson, M. Elad, and P. Milanfar, "Fast and robust multiframe super-resolution," IEEE Trans. Image Process. 13(10), 1327-1344 (2004). 
8. H. He and L. P. Kondi, "Choice of threshold of the Huber-Markov prior in map-based video resolution enhancement," in Canadian Conf. Electrical and Computer Engineering, Vol. 2, pp. 801-804 (2004).

9. P. P. B. Eggermont, "Maximum entropy regularization for Fredholm integral equations of the first kind," SIAM J. Math. Anal. 24(6), $1557-1576$ (1993).

10. L. C. Pickup, S. J. Roberts, and A. Zisserman, "A sampled texture prior for image super-resolution," in Adv. Neural Information Processing Systems Conf., pp. 1587-1594 (2004).

11. V. Patanavijit and S. Jitapunkul, "A robust iterative multiframe superresolution reconstruction using a Huber-Bayesian approach with Huber-Tikhonov regularization," in Int. Symp. on Intelligent Signal Processing and Communications Systems (ISPACS'06), pp. 13-16 (2006).

12. V. Patanavijit and S. Jitapunkul, "An iterative super-resolution reconstruction of image sequences using a Bayesian approach with BTV prior and affine block-based registration," in Proc. 3rd Canadian Conf. Computer and Robot Vision, Vol. 00, p. 45 (2006).

13. V. Patanavijit and S. Jitapunkul, "An iterative super-resolution reconstruction of image sequences using a Bayesian approach and affine block-based registration," in European Signal Processing Conf. (EUSIPCO 2006), Florence, Italy, Sept. 4-8 (2006).

14. V. Patanavijit and S. Jitapunkul, "An iterative super-resolution reconstruction of image sequences using affine block-based registration," in Proc. 2006 Int. Conf. Wireless Commun. Mobile Comput., pp. 5156. ACM, New York (2006).

15. V. Patanavijit, P. Sermwuthisarn, and S. Jitapunkul, "A robust iterative super-resolution reconstruction of image sequences using a Tukey's biweight Bayesian approach with fast affine block-based registration," in IEEE Int. Conf. Multimedia and Expo, pp. 480-483 (2007).

16. V. Patanavijit and S. Jitapunkul, "A Lorentzian stochastic estimation for a robust iterative multiframe super-resolution reconstruction with Lorentzian-Tikhonov regularization," EURASIP J. Adv. Signal Process., 2007, 1-21 (2007)

17. A. Panagiotopoulou and V. Anastassopoulos, "Super-resolution reconstruction of thermal infrared images," in Proc. 4th WSEAS Int. Conf. Remote Sensing, pp. 40-44 (2008).

18. G. Roussas, A First Course in Mathematical Statistics, AddisonWesley, Boston, MA (1973).

19. P. J. Huber, Robust Statistics, John Wiley \& Sons, New York (1981)

20. J. Tukey, Understanding Robust and Exploratory Data Analysis, John Wiley and Sons, New York (1983).

21. M. J. Black, G. Sapiro, D. H. Marimont, and D. Heeger, "Robust anisotropic diffusion," IEEE Trans. Image Process. 7(3), 421-432 (1998).

22. S. Farsiu, D. Robinson, M. Elad, and P. Milanfar, "Advances and challenges in super-resolution," Int. J. Imaging Syst. Technol. 14(2), 47-57 (2004).
23. H. W. Engl, M. Hanke, and A. Neubauer, "Regularization of Inverse Problems," Kluwer Academic Publishers, Dordrecht, The Netherlands (2000).

24. T. Chan, S. Osher, and J. Shen, "The digital TV filter and nonlinear denoising," IEEE Trans. Image Process. 10(2), 231-241 (2001).

25. M. Elad, "On the origin of the bilateral filter and ways to improve it," IEEE Trans. Image Process. 11(10), 1141-1151 (2002).

26. C. S. Xydeas and V. Petrovic, "Objective image fusion performance measure," Electron. Lett. 36(4), 308-309 (2000).

27. http://commons.wikimedia.org/wiki/

File:Stanford_University_Main_Quad_western_archway.JPG\#file.

28. MDSP resolution enhancement software, 2004. (We were licensed to use the software for research purposes).

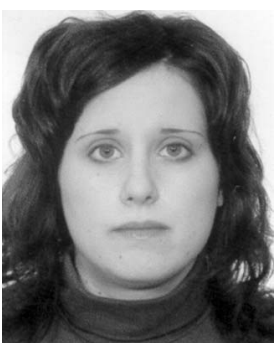

Antigoni Panagiotopoulou received a BSc degree in physics in 2002 and an MSc degree in electronics in 2004 from the Department of Physics, University of Patras, Greece. She is now pursuing a $\mathrm{PhD}$ degree in image resolution improvement in the Department of Physics, University of Patras. Her main research interests focus on resolution enhancement of images, including image interpolation, super-resolution image reconstruction, and neural networks as a tool for constructing a high-resolution image.

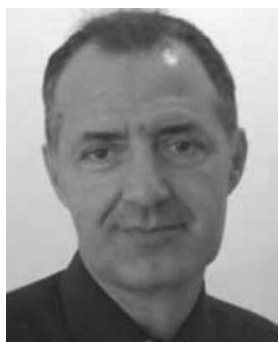

Vassilis Anastassopoulos received a BSc degree in physics in 1980 and his $\mathrm{PhD}$ in electronics in 1986, both from the University of Patras, Greece. From 1985 to 1987, he served in the Greek Army. From 1987 to 1989 , he was a lecturer at the University of Patras. From 1989 to 1990 , he worked as a research associate in the Department of Electrical Engineering, University of Toronto. From 1990 to 1991, he worked as a lecturer in the Electronics Laboratory, University of Patras, Greece. He was promoted to assistant professor in the same university in 1992, to associate professor in 2000, and then to professor in 2005. His research interests are within the scope of digital signal processing, image processing, radar signal processing and remote sensing, and pattern recognition and classification. He is an IEEE member. 